\title{
Gene expression analyses reveal a relationship between conidiation and cerato-platanin in homokaryotic and heterokaryotic strains of the fungal plant pathogen Heterobasidion irregulare
}

\author{
Ivan Baccelli ${ }^{1 *}$, Paolo Gonthier ${ }^{2}$, Rodolfo Bernardi ${ }^{3}$
}

${ }^{1}$ Department of Agri-Food Production and Environmental Sciences, University of Florence, Piazzale delle Cascine 28, 50144, Firenze, Italy; ${ }^{2}$ Department of Agricultural, Forest and Food Sciences, University of Turin, Largo Paolo Braccini 2, 10095, Grugliasco, Italy; ${ }^{3}$ Department of Agriculture, Food and Environment, University of Pisa, Via del Borghetto 80, 56124, Pisa, Italy;

*E-mail: ivan.baccelli@unifi.it (for correspondence)

\begin{abstract}
The Basidiomycete Heterobasidion irregulare was recently sequenced and three cerato-platanin encoding genes were found in its genome (HiCPs). Cerato-platanin family proteins (CPPs) are produced by both plant pathogenic and nonpathogenic fungi, and can act both as virulence factors and elicitors of defence responses. In fungal life these proteins seem to play a dual role, in the fungal cell wall and in the fungus-plant interaction, but most data available to date on CPPs derive from studies performed on Ascomycetes. In the present study, we investigated the expression of $\mathrm{HiCPs}$ in three homokaryotic isolates and two heterokaryotic isolates of the forest pathogen $H$. irregulare. Transcription of $\mathrm{HiCPS}$ was analysed both at the edge and at the centre of the fungal colony and compared between homokaryon and heterokaryon. Results showed that only $\mathrm{HiCP} 1$ and $\mathrm{HiCP} 2$ are likely to be translated in $\mathrm{H}$. irregulare and that, under the tested conditions, $\mathrm{HiCPl}$ is by far the gene with the highest transcript abundance among $\mathrm{HiCPs}$. HiCPl did not show any preferential expression in different sections of the fungal colony, while $\mathrm{HiCP} 2$ was significantly more expressed at the colony centre, thus suggesting a link with the production of conidia. The level of expression of $H i C P s$ in heterokaryons was generally comparable to that of one or both the parental homokaryons, irrespective of the colony section, thus demonstrating that HiCPs are not transcriptionally influenced by the heterokaryotic stage.
\end{abstract}

Keywords: MAMP, PAMP, snodprot, eliciting plant response, small protein, expansins 


\section{Introduction}

Heterobasidion irregulare (Underw.) Garbel. \& Otrosina is a Basidiomycete included in the species complex of $H$. annosum (Fr.) Bref. sensu lato (Garbelotto and Gonthier 2013). H. irregulare is a forest pathogen which, in North America, attacks pine (Pinus spp.), juniper (Juniperus spp.), and incense cedar (Calocedrus decurrens) (Garbelotto and Gonthier 2013). In Italy, where it was introduced during World War II (Garbelotto et al. 2013), the fungus has become invasive in Italian stone pine (Pinus pinea) stands (Gonthier et al. 2007). In the invasion area in Italy, H. irregulare is significantly more widespread than its congener H. annosum sensu stricto (Gonthier et al. 2007, 2014). The two species hybridise, and it was reported that a massive allele introgression mostly occurs unilaterally from the native species to the invasive one (Gonthier and Garbelotto 2011).

In nature, homokaryotic haploid mycelia are responsible for primary infections occurring on the surface of fresh stumps or wounds on the trees, while secondary mycelia, consisting of a mosaic of haploid and heterokaryotic mycelia, may be responsible for the infections occurring from stump to tree or from tree to tree, through root contacts or grafts (Stenlid and Rayner 1991; Garbelotto and Gonthier 2013).

H. irregulare is a wood decay agent that can switch from a saprotrophic lifestyle on stumps to a necrotrophic parasitism on living trees (Olson et al. 2012), and is able to degrade all components of wood, including lignin and cellulose (Yakovlev et al. 2012, 2013; Raffaello et al. 2013). Recently, with the aim of investigating the molecular bases of the two trophic strategies, the genome of $H$. irregulare has been sequenced and, for the first time, the presence of genes encoding cerato-platanin family proteins (CPPs) has been reported (Olson et al. 2012). In that study, the authors included $C P P$ genes among the pathogenicity factors of the fungus.

CPPs are an enigmatic family of proteins found exclusively in filamentous fungi that seem to actually play a role in virulence for some pathogens (Jeong et al. 2007; Frías et al. 2011). However, most studies have investigated the ability of CPPs to act as elicitors of defence responses when separately applied to plants (Djonović et al. 2006; Yang et al. 2009; Frías et al. 2013; Baccelli et al. 2014a). Fungi abundantly secrete CPPs into their culture media, but some studies have also localised these proteins within the fungal cell wall (Gaderer et al. 2014; Pazzagli et al. 2014). As recently demonstrated, CPPs are not hydrophobin-like proteins, because they have different biochemical and structural properties (de Oliveira et al. 2011; de O. Barsottini et al. 2013; Frischmann et al. 2013). CPPs are instead partially similar to proteins named expansins, which in plants mediate cell wall loosening and are involved in various processes like growth (Sampedro and Cosgrove 2005; Baccelli et al. 2014b). On the basis of the results obtained up to date, it seems plausible that, in fungal life, CPPs may act in an expansin-like manner and cause the loosening of both fungal and plant cell walls (Baccelli 2015). Ascomycetes usually have one or two CPP encoding genes and most information available to date on CPPs comes from these fungi (Chen et al. 2013; Gaderer et al. 2014). Basidiomycetes may have up to twelve CPP genes, but only those from Moniliophthora perniciosa have been studied up to date (Chen et al. 2013; de O. Barsottini et al. 2013).

In the present study, we aimed at investigating the transcriptional regulation of $C P P s$ in the Basidiomycete $H$. irregulare, which harbours three CPP encoding genes in its genome (Olson et al. 2012). We analysed and compared the transcription of CPPs in five isolates, three homokaryotic isolates and two heterokaryotic isolates. Homokaryotic and heterokaryotic mycelia not only play different roles in the infectious process, but also differ in their biology: clamp connections are formed in the heterokaryotic mycelia. As CPPs have been found localised in the fungal cell wall and may act as expansins, we hypothesised that the expression of $C P P$ genes could be influenced in the heterokaryotic mycelia. In addition, we tested whether $C P P$ genes were differently transcribed within the fungal colony in relation to other morphological or physiological features.

\section{Materials and methods}

Fungal strains and culture conditions

Three homokaryotic haploid isolates and two heterokaryotic isolates of $H$. irregulare were used in this study. Homokaryotic isolates 142EF, 53OA and 89EG were obtained from spores landed on woody spore traps exposed in the Circeo National Park (Sabaudia, Italy), Gallinara pinewood (Anzio, Italy), and La Campana pinewood (Nettuno, Italy), respectively, and were collected and isolated in pure culture as previously described (Gonthier and Garbelotto 2011). The purity of the isolates (over 95\% assignment to $H$. irregulare) was assessed in a study based on the characterisation of over 500 AFLP loci (Gonthier and Garbelotto 2011). The two heterokaryotic isolates 142x53OA and 142x89EG were obtained in the laboratory by mating the isolates $142 \mathrm{EF}$ with $53 \mathrm{OA}$, and 142EF with $89 \mathrm{EG}$, respectively. Inocula of the clampless parental homokaryotic isolates were placed about $1 \mathrm{~cm}$ apart in the middle of a 9-cm diameter Petri dish filled with $1.5 \%$ malt extract agar (MEA) (Difco, Detroit, MI). After 3 weeks, a small piece of mycelium was taken from the zone of contact of the two isolates and transferred into a new Petri dish, resulting in a heterokaryotic isolate as assessed under 200x magnification for the presence of clamp connections. 
The isolates were grown and maintained in 9-cm diameter Petri dishes on MEA at $23{ }^{\circ} \mathrm{C}$, in the dark. In order to facilitate removal of mycelium for the subsequent DNA and RNA extractions, the isolates were grown on a cellophane disc placed on the surface of the culture medium. Cellophane discs were sterilised in autoclave at $121^{\circ} \mathrm{C}$ for $15 \mathrm{~min}$.

DNA extraction and sequencing of cerato-platanin encoding genes

DNA was obtained from the homokaryotic haploid isolates by grinding $30-50 \mathrm{mg}$ of mycelium in $200 \mu \mathrm{l}$ of Hexadecyltrimethylammonium bromide (CTAB) lysis buffer ( $\mathrm{NaCl} 1.4 \mathrm{M}$; EDTA $20 \mathrm{mM}$; Tris-HCl $100 \mathrm{mM}$, pH 8.0; CTAB 3\% w/v; 2-Mercaptoethanol $0.2 \% \mathrm{v} / \mathrm{v})$. DNA was then extracted with isoamyl alcohol-chloroform and precipitated with isopropyl alcohol.

The coding regions of the CPP genes were amplified with PCR by using primers designed on the basis of the sequences and annotations present in JGI MycoCosm (Fungal Genomic Resource, Joint Genome Institute) for the sequenced strain TC32-1 of $H$. irregulare (Olson et al. 2012). Both forward and reverse primers were designed on the untranslated regions (UTRs) 5' and 3'.

The following primers were designed and used (HiCP stands for H. irregulare cerato-platanin): HiCPl Forward (For) 5'-GTGCTCTCATCTCTGTCGTCC, Reverse (Rev) 5'-TCCAAAGCGTAACGATCTTCCT; HiCP2 For 5'CACCAATTCATTGCATTTACACACT, Rev 5'-AGGCTCGTGCATACATGTGAA; HiCP3 For 5'CTCACACTCGACTAGCGCAT, Rev 5'-TGGAACACTTGTATCTCACCAT. In addition, HiCP2 sequences were further extended at the 5'-end by using the primers For 5'-GGACAGCCATATCTTCCGACACC and Rev 5'GGGACGTAGATGGTTTGGACC.

All the reactions were carried out with $2.5 \mathrm{mM} \mathrm{MgCl}_{2}, 200 \mu \mathrm{M}$ dNTPs, $400 \mathrm{nM}$ primer (each), 1X Reaction Buffer, and $0.05 \mathrm{U} \mathrm{\mu l}^{-1}$ EuroTaq DNA polymerase (Euro Clone, Italy), at an annealing temperature of $57{ }^{\circ} \mathrm{C}$ for $28-30$ cycles.

The reaction products were purified from electrophoresis gel with Wizard SV Gel and PCR Clean-Up System (Promega Italia Srl, Italy) and sequenced by Eurofins MWG Operon/Carlo Erba Reagents Srl, Italy. In order to avoid the presence of amplification mistakes within the sequence, both amplification reactions and sequencing were repeated.

The sequences obtained were analysed with on-line tools such as ClustalW, ExPASy and BLAST software, and finally deposited in GenBank under the accession numbers LN626599-LN626607. Signal peptide predictions were performed with PrediSi.

\section{Growth rates}

The radial growth rate of each isolate was determined on MEA in 9-cm Petri dishes, at $23{ }^{\circ} \mathrm{C}$, in the dark, by growing the fungus on a cellophane disc for the subsequent RNA extraction. The Petri dishes were inoculated in the centre with an 8-mm diameter agar plug obtained from the edge of actively growing fungal cultures. The radial growth was measured daily along two perpendicular lines during 6 days. Five replicates were grown per each isolate and the experiment was repeated three times over a period of 3 months.

\section{Microscopic analysis}

Microscopical observations of conidial presence were performed both from the edge (last 24-h growth) and from the centre of the colony (48-144 h), after differentiating mycelium sections as described in the following paragraph. The production of conidia and conidiophores was determined by examining 5 field of view (FOV) at 200x magnification on three experimental replicates obtained as described above. The production of conidiophores was expressed as number per FOV, whereas the production of conidia was expressed as,+++ , or +++ depending on the relative abundance of dispersed conidia found in the samples.

\section{RNA extraction and transcription analysis}

Mycelium of each isolate was collected from the cellophane layer of a randomly selected culture by respecting the following scheme: from the edge of the colony it was collected the mycelium grown in the last $24 \mathrm{~h}$ (i.e. $0-24 \mathrm{~h}$ old); the mycelium 24-48 h old was left on the plate and considered as an intermediate zone; the mycelium in the centre of the colony was collected from the intermediate zone up to the edge of the agar plug (i.e. $48-144 \mathrm{~h}$ old). The intermediate zone was not subjected to further analysis with the aim of enhancing possible differences in the level of expression between the mycelium sections.

Total RNA was isolated from mycelium with RNeasy Plant Mini Kit (Qiagen, CA) by using RLT buffer. RNA was treated with DNase by using Amplification Grade DNase I (Sigma-Aldrich, MO) and reverse-transcribed (400 ng per sample) into cDNA with iScript cDNA synthesis kit (BioRad, USA). 
Real-time qPCR reactions $(20 \mu \mathrm{l})$ were carried out with $10 \mathrm{ng}$ of cDNA, $250 \mathrm{nM}$ primers, and 1x Fast SYBR Green Master Mix (Applied Biosystems, CA) following the manufacturer's instructions. PCRs were run in a StepOne realtime PCR System (Applied Biosystems) by using the recommended thermal-cycling conditions (hold $95{ }^{\circ} \mathrm{C}, 20 \mathrm{~s} ; 40$ cycles $95^{\circ} \mathrm{C}, 3 \mathrm{~s} ; 60^{\circ} \mathrm{C}, 30 \mathrm{~s}$ ). Gene specific primers for $\mathrm{HiCP} 1, \mathrm{HiCP} 2$ and $\mathrm{HiCP} 3$ were designed with Primer Express Software 3.0 (Applied Biosystems) so that the allelic variants resulted from the previous sequencing could not be distinguished. The following primers were designed and used: HiCP1 For 5'-CACGAACGGCCTCATCAAC, Rev 5'GACGTTCGGGAAAGACGGTAA; HiCP2 For 5'-CCCGACCTTCAGCGATCTAC, Rev 5'ACCCGACGGCGAAAGC; HiCP3 For 5'-CAGTTCTACGCCAAGTGCCTACT, Rev 5'GACGTGCCGCTGGGATAA.

Relative gene expression values $\left(2^{-\Delta \Delta \mathrm{Ct}}\right)$ were calculated by using $18 \mathrm{~S}$ rRNA gene as the endogenous reference gene following the calculation described in ABI PRISM 7700 Sequence Detection System User Bulletin \#2 (Applied Biosystems). $18 S$ primers were designed by using the GenBank sequence AF026576 (primers For 5'TGGTGCATGGCCGTTCTT, Rev 5'-AGCAGGTTAAGGTCTCGTTCGT). 18S was used as the reference gene after confirmation of its transcriptional stability across isolates and mycelium sections.

Before the quantification, a validation experiment was performed to ensure that the amplification efficiencies of the target genes and the reference gene were comparable.

\section{Statistical analysis}

Relative gene expression data $\left(2^{-\Delta \Delta C t}\right)$ were analysed with one-sample $t$ test (centre vs. edge $=1$ ) or with one-way ANOVA with Tukey-Kramer post test (heterokaryon vs. parental homokaryons; common parental 142EF=1). Growth rates and production of conidiophores were similarly analysed with one-way ANOVA with Tukey-Kramer post test at $P<0.05$. All the statistical analyses were performed by using GraphPad InStat v. 3.05 (GraphPad Software, San Diego, CA).

\section{Results}

Sequencing of cerato-platanin encoding genes in Italian isolates of Heterobasidion irregulare

The length of $\mathrm{HiCP}$ sequences from the isolates 142EF, 53OA and 89EG was 545 bp for HiCP1, 1032-1035 bp for $\mathrm{HiCP} 2$ and $651 \mathrm{bp}$ for $\mathrm{HiCP} 3$. HiCPl coded for a protein of 138 amino acids, with a putative N-terminal signal peptide of 19 amino acids (Fig. 1), and showed the canonical domain structure of CPPs (Chen et al. 2013). In addition, by sequencing $\mathrm{HiCPl}$ from DNA and cDNA, we confirmed the presence of a 65-bp intron located in the 3'UTR (Online Resource, Fig. S1). HiCP2 coded for a protein of 259-260 amino acids, with a putative signal peptide of 19 amino acids, and was composed of an N-terminal region with many repetitions with no apparent similarity to known proteins, and a C-terminal region which showed similarities with the CPP domain. Finally, HiCP3 seemed to encode for a truncated version of $\mathrm{HiCP} 2$ of 105 amino acids, without a signal peptide, and was apparently contained in a putative longer transcript of about $4 \mathrm{~kb}$ (data not shown).

By comparing the sequences obtained in this study with those available from the American strain TC32-1, we found that the deduced amino acid sequences of $\mathrm{HiCP} 1$ and $\mathrm{HiCP} 3$ were highly conserved (Fig. 1). In fact, all the three homokaryiotic Italian isolates and TC32-1 showed the same amino acid sequence. However, we found five putative single nucleotide polymorphisms (SNPs) in the nucleotide sequence of HiCP1, two in the coding region and three within the intron (Online Resource, Fig. S1). HiCP2 showed instead more polymorphisms, both at the level of gene and protein sequences, including a deletion in the Italian isolate 53OA (Fig. 1 and Online Resource, Fig. S2). HiCP3 showed only one putative SNP (Online Resource, Fig. S3).

\section{Transcription analysis of cerato-platanin genes}

Based on a general analysis of the qPCR data, $H i C P 1$ always turned out to be the $C P P$ gene with the highest transcript abundance, with a level of transcription at least 300 fold higher than $\mathrm{HiCP} 2$ or $\mathrm{HiCP} 3$; HiCP2 was generally more transcribed than $\mathrm{HiCP} 3$ (Online Resource, Table S1).

When mycelium collected from the centre of the colony (48-144 h old) was compared to mycelium collected from actively growing hyphae ( $0-24 \mathrm{~h}$ old, edge of the colony), no significant difference in the relative level of transcription was found for $\mathrm{HiCPl}$ (Fig. 2). Interestingly, when $\mathrm{HiCP} 2$ was analysed, it was significantly more expressed at the centre of the colony, and this was true in all isolates. The transcription level of HiCP3 was not significantly different in the two tested colony sections, although the isolate 142EF showed a high transcription level in the centre of the colony. The heterokaryon 142EFx53OA showed, for $\mathrm{HiCP}$, the same level of transcription as the parental homokaryons in both tested colony sections (Fig. 3A); for HiCP2 and HiCP3 it showed instead differences compared to the homokaryon 
142EF, either in the colony centre $(\mathrm{HiCP} 2)$ or in both the sections ( $\mathrm{HiCP} 3)$, but no significant differences were observed between the heterokaryon and the other parental homokaryon 53OA (Fig. 3A). Concerning the combination 142EF with 89EG (Fig. 3B), although $\mathrm{HiCPl}$ was slightly more expressed in the centre of the colony at the heterokaryotic stage, in all the other cases the level of expression of the $C P P$ genes in the heterokaryon was comparable to that of one or both the parental homokaryotic isolates.

\section{Morphological analysis of the isolates}

Growth rate and production of conidia and conidiophores were analysed in order to find possible relationships with the expression pattern of the CPP genes. Neither conidia nor conidiophores were observed at the edge of the colony, i.e. the younger part of the mycelium; on the contrary, all the strains abundantly produced conidia and conidiophores at the centre. The heterokaryotic isolates released less conidia than the parental homokaryotic isolates, but the isolates did not statistically differ in the production of conidiophores (Table 1).

The average radial growth rates ranged from $4.5 \mathrm{~mm} \mathrm{day}^{-1}$ of the isolate $142 \mathrm{EF}$ to $6.2 \mathrm{~mm} \mathrm{day}^{-1}$ of the isolate $89 \mathrm{EG}$ (Fig. 4). With the only exception represented by this comparison, differences in the growth rate between isolates were not significant.

\section{Discussion}

CPPs have been found in more than 50 fungal genomes, and Basidiomycetes show both the higher sequence diversity and number of homologs (Chen et al. 2013). However, the current knowledge concerning CPPs almost exclusively derives from studies performed on Ascomycetes. The hemibiotroph Moniliophthora perniciosa, the causal agent of witches' broom disease in cacao, is the only Basidiomycete where CPPs have been studied so far (de O. Barsottini et al. 2013). In the present study, we investigated the regulation of CPPs in a necrotrophic Basidiomycete, the conifer root rot pathogen $H$. irregulare, and obtained for the first time data on how these genes are transcribed within the fungal colony at the homokaryotic and heterokaryotic stage.

Based on the sequencing and annotation of the North American H. irregulare strain TC32-1, we identified three $C P P$ encoding genes: CerPla1, CerPla2 and CerPla3 (Olson et al. 2012). However, in order to follow the current denomination of CPPs and to differentiate them clearly from cerato-platanin from $C$. platani, here we propose to rename these genes as HiCP1 (H. irregulare cerato-platanin 1 ), HiCP2 and HiCP3.

The sequence analysis showed that $H$. irregulare possesses three highly different CPPs, with a length of 138 amino acids (HiCP1), 259-260 amino acids (HiCP2) and 105 amino acids (HiCP3). Thus, this analysis firstly allowed correction of the previous sequence annotations: six terminal amino acids were added to HiCP2; a putative start codon was identified in HiCP3.

HiCP1 was the only HiCP with the canonical structure of CPPs, i.e. typical length with signal peptide (Chen et al. 2013), and was also the gene showing the highest transcript abundance. HiCP2 and HiCP3 were both transcribed as well, but to a lower extent. Nevertheless, the translation into a protein of $\mathrm{HiCP} 3$ seemed unlikely for several reasons: it appeared as a truncated version of $\mathrm{HiCP}$, the localisation of the start codon was accordingly uncertain, a signal peptide could not be found, and the length of the protein sequence was the shortest ever reported so far for CPPs (Chen et al. 2013).

The transcriptional study was performed on five isolates which were very similar in their growth rate. Thus, we did not attempt to compare the transcription level of HiCPs between isolates to find correlations with their growth rate. We studied instead the transcription of HiCPs within each colony, by comparing the edge with the centre.

We found that $\mathrm{HiCPl}$ was transcribed by the isolates without significant differences between colony sections, while HiCP2 showed a clear expression pattern: it was significantly more transcribed at the centre of the colony, and this was true in all isolates. The edge and the centre of the colony are two clearly different physiological and morphological zones: at the edge of the colony the fungus has actively growing hyphae lacking conidiophores and conidia, while the colony centre represents the older part with abundant conidia. Therefore, this result may suggest a link between $\mathrm{HiCP} 2$ and the production of conidia, according to the evidence of a functional diversification and specialisation of CPP homologs (de O. Barsottini et al. 2013; Frischmann et al. 2013). It is also interesting to note that, although CPPs do not possess the biochemical properties of hydrophobins (Frischmann et al. 2013), the gene induction during conidiation is reminiscent of hydrophobins (Dubey et al. 2014).

No relative increase in the transcription level of HiCPs was found in the heterokaryotic mycelia. The level of expression in heterokaryons was generally comparable to that of one or both the parental homokaryons, irrespective of the colony section, thus demonstrating that HiCPs are not transcriptionally influenced by the heterokaryotic stage.

In conclusion, this study has shown for the first time that $C P P$ genes are differently transcribed within the fungal colony, and this occurs in both homokaryotic and heterokaryotic mycelia. However, their expression level is not altered by heterokaryosis. Our findings suggest that only $\mathrm{HiCP} 1$ and $\mathrm{HiCP} 2$ are likely to play a role in the biology of $\mathrm{H}$. irregulare, with $\mathrm{HiCPl}$ having probably the major role. In fact, HiCPl showed the highest transcript abundance in all isolates and it did not show preferential expression in different sections of the colony. Further studies will help to understand whether $\mathrm{HiCPl}$ can play a role in both hyphal elongation and the production of conidia, by acting as 
expansin-like protein in the cell wall as similarly suggested for CP from C. platani (Baccelli et al. 2012; Baccelli 2015), and whether $H i C P 2$ actually has a preferential role during the formation of conidia, similarly to the $C P P$ gene epl2 from Trichoderma atroviride (Frischmann et al. 2013).

\section{Acknowledgements}

This work was supported by Fondi di Ateneo year 2012-2013, Università di Pisa, to RB. Partial support was also obtained by the Italian Ministry of Education, University and Research, within the FIRB program (grant number RBFRI280NN) to PG. IB was supported by a grant from Ente Cassa di Risparmio di Firenze (No. 2013/0444). The authors thank Dr. Luana Giordano for help with the laboratory work.

\section{Conflict of Interest}

The authors declare that they have no conflict of interest

\section{References}

Baccelli I, Comparini C, Bettini PP, Martellini F, Ruocco M, Pazzagli L, Bernardi R, Scala A (2012) The expression of the cerato-platanin gene is related to hyphal growth and chlamydospores formation in Ceratocystis platani. FEMS Microbiol Lett 327:155-163

Baccelli I, Lombardi L, Luti S, Bernardi R, Picciarelli P, Scala A, Pazzagli L (2014a) Cerato-platanin induces resistance in Arabidopsis leaves through stomatal perception, overexpression of salicylic acid- and ethylene-signalling genes and camalexin biosynthesis. PLoS One 9:e100959

Baccelli I, Luti S, Bernardi R, Scala A, Pazzagli L (2014b) Cerato-platanin shows expansin-like activity on cellulosic materials. Appl Microbiol Biotechnol 98:175-184

Baccelli I (2015) Cerato-platanin family proteins: one function for multiple biological roles?. Front Plant Sci 5:769

Chen H, Kovalchuk A, Keriö S, Asiegbu FO (2013) Distribution and bioinformatic analysis of the cerato-platanin protein family in Dikarya. Mycologia 105:1479-1488

de O Barsottini MR, de Oliveira JF, Adamoski D, Teixeira PJ, do Prado PF, Tiezzi HO, Sforça ML, Cassago A, Portugal RV, de Oliveira PS, de M Zeri AC, Dias SM, Pereira GA, Ambrosio AL (2013) Functional diversification of cerato-platanins in Moniliophthora perniciosa as seen by differential expression and protein function specialization. Mol Plant Microbe Interact 26:1281-1293

de Oliveira AL, Gallo M, Pazzagli L, Benedetti CE, Cappugi G, Scala A, Pantera B, Spisni A, Pertinhez TA, Cicero DO (2011) The structure of the elicitor Cerato-platanin (CP), the first member of the CP fungal protein family, reveals a double $\psi \beta$-barrel fold and carbohydrate binding. J Biol Chem 286:17560-17568

Djonović S, Pozo MJ, Dangott LJ, Howell CR, Kenerley CM (2006) Sm1, a proteinaceous elicitor secreted by the biocontrol fungus Trichoderma virens induces plant defense responses and systemic resistance. Mol Plant Microbe Interact 19:838-853

Dubey MK, Jensen DF, Karlsson M (2014) Hydrophobins are required for conidial hydrophobicity and plant root colonization in the fungal biocontrol agent Clonostachys rosea. BMC Microbiol 14:18

Frías M, Brito N, González C (2013) The Botrytis cinerea cerato-platanin BcSpl1 is a potent inducer of systemic acquired resistance (SAR) in tobacco and generates a wave of salicylic acid expanding from the site of application. Mol Plant Pathol 14:191-196

Frías M, González C, Brito N (2011) BcSpl1, a cerato-platanin family protein, contributes to Botrytis cinerea virulence and elicits the hypersensitive response in the host. New Phytol 192:483-495

Frischmann A, Neudl S, Gaderer R, Bonazza K, Zach S, Gruber S, Spadiut O, Friedbacher G, Grothe H, Seidl-Seiboth V (2013) Self-assembly at air/water interfaces and carbohydrate binding properties of the small secreted protein EPL1 from the fungus Trichoderma atroviride. J Biol Chem 288:4278-4287

Gaderer R, Bonazza K, Seidl-Seiboth V (2014) Cerato-platanins: a fungal protein family with intriguing properties and application potential. Appl Microbiol Biotechnol 98:4795-4803

Garbelotto M, Gonthier P (2013) Biology, epidemiology, and control of Heterobasidion species worldwide. Annu Rev Phytopathol 51:39-59

Garbelotto M, Guglielmo F, Mascheretti S, Croucher PJ, Gonthier P (2013) Population genetic analyses provide insights on the introduction pathway and spread patterns of the North American forest pathogen Heterobasidion irregulare in Italy. Mol Ecol 22:4855-4869

Gonthier P, Anselmi N, Capretti P, Bussotti F, Feducci M, Giordano L, Honorati T, Lione G, Luchi N, Michelozzi M, Paparatti B, Sillo F, Vettraino AM, Garbelotto M (2014) An integrated approach to control the introduced forest pathogen Heterobasidion irregulare in Europe. Forestry 87:471-481 
Gonthier P, Garbelotto M (2011) Amplified fragment length polymorphism and sequence analyses reveal massive gene introgression from the European fungal pathogen Heterobasidion annosum into its introduced congener $H$. irregulare. Mol Ecol 20:2756-2770

Gonthier P, Nicolotti G, Linzer R, Guglielmo F, Garbelotto M (2007) Invasion of European pine stands by a North American forest pathogen and its hybridization with a native interfertile taxon. Mol Ecol 16:1389-1400

Jeong JS, Mitchell TK, Dean RA (2007) The Magnaporthe grisea snodprot1 homolog, MSP1, is required for virulence. FEMS Microbiol Lett 273:157-165

Olson A, Aerts A, Asiegbu F, Belbahri L, Bouzid O, Broberg A, Canbäck B, Coutinho PM, Cullen D, Dalman K, Deflorio G, van Diepen LT, Dunand C, Duplessis S, Durling M, Gonthier P, Grimwood J, Fossdal CG, Hansson D, Henrissat B, Hietala A, Himmelstrand K, Hoffmeister D, Högberg N, James TY, Karlsson M, Kohler A, Kües U, Lee YH, Lin YC, Lind M, Lindquist E, Lombard V, Lucas S, Lundén K, Morin E, Murat C, Park J, Raffaello T, Rouzé P, Salamov A, Schmutz J, Solheim H, Ståhlberg J, Vélëz H, de Vries RP, Wiebenga A, Woodward S, Yakovlev I, Garbelotto M, Martin F, Grigoriev IV, Stenlid J (2012) Insight into trade-off between wood decay and parasitism from the genome of a fungal forest pathogen. New Phytol 194:1001-1013

Pazzagli L, Seidl-Seiboth V, Barsottini M, Vargas WA, Scala A, Mukherjee PK (2014) Cerato-platanins: elicitors and effectors. Plant Sci 228:79-87

Raffaello T, Chen H, Kohler A, Asiegbu FO (2013) Transcriptomic profiles of Heterobasidion annosum under abiotic stresses and during saprotrophic growth in bark, sapwood and heartwood. Environ Microbiol 16:1654-1667

Sampedro J, Cosgrove DJ (2005) The expansin superfamily. Genome Biol 6:242

Stenlid J, Rayner ADM (1991) Patterns of nuclear migration and heterokaryosis in pairings between sibling homokaryons of Heterobasidion annosum. Mycol Res 95:1275-1283

Yakovlev I, Vaaje-Kolstad G, Hietala AM, Stefańczyk E, Solheim H, Fossdal CG (2012) Substrate-specific transcription of the enigmatic GH61 family of the pathogenic white-rot fungus Heterobasidion irregulare during growth on lignocellulose. Appl Microbiol Biotechnol 95:979-990

Yakovlev IA, Hietala AM, Courty PE, Lundell T, Solheim H, Fossdal CG (2013) Genes associated with lignin degradation in the polyphagous white-rot pathogen Heterobasidion irregulare show substrate-specific regulation. Fungal Genet Biol 56:17-24

Yang Y, Zhang H, Li G, Li W, Wang X, Song F (2009) Ectopic expression of MgSM1, a Cerato-platanin family protein from Magnaporthe grisea, confers broad-spectrum disease resistance in Arabidopsis. Plant Biotechnol J 7:763-777

\section{Supplementary Material}

Fig. S1 ClustalW alignment of $H i C P 1$ nucleotide sequences

Fig. S2 ClustalW alignment of $\mathrm{HiCP} 2$ nucleotide sequences

Fig. S3 ClustalW alignment of $\mathrm{HiCP} 3$ nucleotide sequences

Table S1 Average $\Delta \mathrm{Ct}$ values

Table 1 Production of conidia and conidiophores in different zones of mycelium

\begin{tabular}{lcccc}
\hline & \multicolumn{2}{c}{ Centre of the colony $^{\mathrm{a}}$} & \multicolumn{2}{c}{ Edge of the colony $^{\mathrm{b}}$} \\
\cline { 2 - 5 } Isolate & Conidiophores $^{\mathrm{c}}$ & Conidia $^{\mathrm{d}}$ & Conidiophores $^{\text {Conidia }^{2}}$ & n.o. \\
\hline 53OA & $6.6 \pm 5.9$ (a) & +++ & n.o. & n.o. \\
89EG & $7.2 \pm 4.3$ (a) & +++ & n.o. & n.o. \\
$142 \mathrm{EF}$ & $3.9 \pm 0.5$ (a) & +++ & n.o. & n.o. \\
$142 \mathrm{EFx} 53 \mathrm{OA}$ & $3.5 \pm 1.8(\mathrm{a})$ & ++ & n.o. & n.o.
\end{tabular}

\footnotetext{
${ }^{\mathrm{a}}$ Mycelium 48-144 h old.

${ }^{\mathrm{b}}$ Mycelium 0-24 h old.

${ }^{\mathrm{c}}$ Conidiophores are expressed as average number \pm SD per field of view (FOV) at 200x; n.o., not observed.

${ }^{\mathrm{d}}$ Dispersed conidia are expressed as $(++)$ or $(+++)$ depending on the relative abundance in the strain samples; n.o., not observed.

Five FOVs per replicate were examined. Data represent three replicates. Statistical analysis was performed with oneway ANOVA with Tukey-Kramer post test at $P<0.05$.
} 


\section{Figure legends}

Fig. 1 Amino acid alignment of Heterobasidion irregulare cerato-platanins (HiCPs). Deduced amino acid sequences were aligned with ClustalW. Italian homokaryotic isolates (142EF, 89EG, 53OA) were aligned with the American strain TC32-1. The annotations in MycoCosm for TC32-1 were reconsidered after BLASTX analysis by adding six Cterminal amino acids in HiCP2 and by identifying a putative start codon in HiCP3. The cerato-platanin domain is underlined. Conserved cysteines and signature sequences (CSD or CSN) of the family are highlighted (Chen et al. 2013). Boxes indicate the predicted signal peptide. Asterisks indicate invariable residues

Fig. 2 Relative expression of cerato-platanin encoding genes ( $\mathrm{HiCPs}$ ) within the fungal colony (centre vs. edge). The analysis was performed in five isolates of Heterobasidion irregulare, three homokaryons (142EF, 89EG, 53OA) and two heterokaryons (142EFx53OA, 142EFx89EG). Relative gene expression values were determined with real time RTPCR by comparing mycelium collected from the centre of the colony ( $48-144 \mathrm{~h}$ old) to mycelium collected from the colony edge ( $0-24 \mathrm{~h}$ old $)$, which was used as the calibrator in the analysis $\left(2^{-\Delta \Delta \mathrm{Ct}}\right.$ or fold change value $\left.=1\right)$. Average fold change values \pm SD from three biological replicates are shown. Statistical analysis was performed with one-sample $t$ test. Asterisk indicates significantly different at $P<0.05$

Fig. 3 Relative expression of cerato-platanin genes (HiCPs) in homokaryotic and heterokaryotic mycelia. The heterokaryons 142EFx530A (a) and 142EFx89EG (b) were analysed in comparison to the respective parental homokaryons. The analysis was performed both at the colony centre and at the colony edge. Relative gene expression values were determined with real time RT-PCR by using the common parental 142EF as the calibrator in the analysis $\left(2^{-\Delta \Delta \mathrm{Ct}}\right.$ or fold change value $\left.=1\right)$. Average fold change values \pm SD from three biological replicates are shown. Statistical analysis was performed with one-way ANOVA with Tukey-Kramer post test at $P<0.05$

Fig. 4 Radial growth rate of the isolates of Heterobasidion irregulare used in the present study. The isolates were grown on malt extract agar, at $23{ }^{\circ} \mathrm{C}$, for 6 days. Five replicates were grown per each isolate and the experiment was repeated three times over a period of three months. Average data with SD are shown. Statistical analysis was performed with one-way ANOVA with Tukey-Kramer post test at $P<0.05$ 
Fig.1

HiCP1

TC32-1

$142 \mathrm{EF}$

$89 E G$

$530 \mathrm{~A}$

TC32-1

$142 \mathrm{EF}$

$89 \mathrm{EG}$

$530 \mathrm{~A}$

TC32-1

$142 \mathrm{EF}$

$89 \mathrm{E} G$

$530 \mathrm{~A}$

\section{$\mathrm{HiCP} 2$}

TC32-1

$142 \mathrm{EF}$

$89 \mathrm{EG}$

$530 \mathrm{~A}$

TC32-1

$142 \mathrm{EF}$

$89 E G$

$530 \mathrm{~A}$

TC32-1

$142 \mathrm{EF}$

$89 \mathrm{EG}$

$53 \mathrm{OA}$

TC32-1

$142 \mathrm{EF}$

$89 \mathrm{EG}$

$530 \mathrm{~A}$

TC32-1

$142 \mathrm{EF}$

$89 \mathrm{EG}$

$530 \mathrm{~A}$

\section{HiCP3}

TC32-1 $142 \mathrm{EF}$ $89 \mathrm{EG}$

$53 \mathrm{OA}$

TC32-1
MKF TF AL ASL AVL AST A A A TDVRYDE TYDN MNEPL TDVACSDGTNGL INKGF STLGSLPS MKF TF AL ASL AVL AST A A ATDVRYDETYDN ANE PL TDV ACSDGTNGL INKGFSTLGSLPS MKF TF AL ASL AVL AST A AF ATDVR YDE TYDN ANEPL TDV ACSDGTNGL INKGF STLGSLPS MKF TF AL ASL AVL AST TAF ATDVRYDE TYDN ANEPL TDVACSDGTNGL INKGFSTLGSLPS

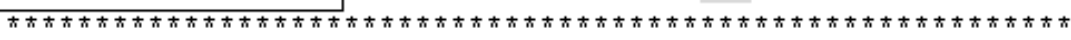

F PNV ÅVQÄ I A GWNSPSCGTCWEVTYNGRSVLVT GVDH H GDG INMSLE AMNTL TNNQGV A F PNV A AVQA I A GWNSPSCGTCWEVTYNGRSVLVTGVD H A GD G INMS LE AMNTL TNNQGVA F PNV A AVQA I A GWNS PSCGTCWEVTYNGRSVLVT GVDH H GD GINMSLE AMNTL TNNQGV A F PNV A AVQA I A GWNSPSCGTCWEVTYNGRSVLVTGVD HAGD G INMS LE AMNTL TNNQGVA

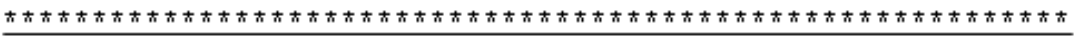

LGTVSATVTQVAMSQCGL LGTVSA AVTOVÄSQCGL LGTVSATVTQVABSQCGL LGTVSATVTQVAMSQCGL

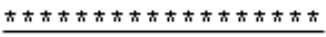

MKFTASF IAVAALFHGTAA APQDGGAPPTPNSPSGTSATSAVTWSKPSTSPVYSSTTSTY MKF TASF IAVALLF HGTA A APQDGGASPTPNSPSGTSATSAVTWSKPSTSPVYSSTTSTY MKF TASF IAVAALF HGTA A APQDGGASPTPNSPSGTSATSAVTUSKPSTSPVYSSTTSTY MKF TASF IAVAMLF HGTAMAMQDGGAPPTPNSPSGTS ATSAVTWSKPSTSPVYSSTTSTY

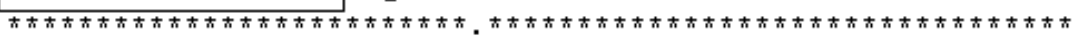

SSTTSTTSPYTTTSATTTWYPSSTSSWYPPSTSSKPYPSSSSYPVPSSSHSYPPPPSGSA SSTTSTTSPYTTTSATTTWYPSSTSSWYPPSTSSKPYPSSSSYPVPSSSHSYPPPPSGSA SSTTSTTSPYTTTSATTTWYPSSTSSWYPPSTSSKPYPSSSSYPVPSSSHSYPPPPSGS A SSTTSTTSPYTT-SATTTWYPSSTSSWYPPSTSSKPYPSSSSYPVPSSSHSYPPPPSGSA

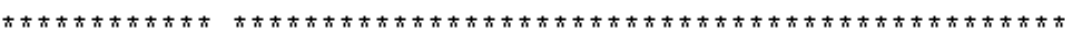

TPPYPSNCPPNPNSNPLMMSLPLTYDNTYDNGSGSMNSVACSNGPKGLVERFPTFSDLPT TPPYPSNCP PNPNSNPL MMSLPL TYDNTYDNGSGSMNSVACSNGP KGLVGRF PTF SDLPT TPPYPSNCPPNPNSNPL MMSLPLTYDNTYDNGSGSMNSV ACSNGP KGLVGRF PTFSDLPT TPPYPSNCP PNPNSNPL MMSLPL TYDNTYDNGSGSMNSVACSNGP KGLVERFPTF SDLPT

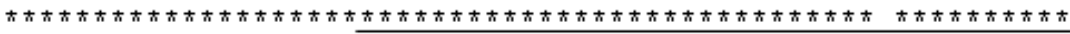

FPY IGGAF AVGSWSSPNCGSCWSL TYPQTGVT IKL I A ID TSGVGFNAMQÄMDKL TGGKA FPY I GGAF AVGSWSSPNCGSCWSL TYPQTGVT IKL I A ID TSGVGFNAMQA AMD KL TGGKA FPY IGGAF AVGSWSSPNCGSCWSL TYPQTGVT IKL I A IDTSGVGFN A AQAMMDKL TGGKA FPY IGGAF AVGSWSSPNCGSCUSL TYPQTGVT IKL I A ID TSGVGFNAMQA AMD KL TGGKA

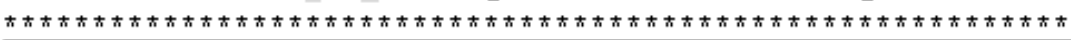

NQLGR IEVNA YQLP ASECKL NQLGR IEVNA YQLP ASECKL NQLGRIEVNA YQLP ASECKL NQLGR IEVNAYQLPASECKL

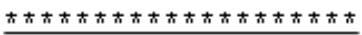

MNTAMCSNGPHGL ASKF P TF GDLPD YPYVGGVF AVSSWNS ANCGTCWAVTYPETGVTINV MNTAMCSNGPHGL ASKF P TF GDLPD YP YVGGVF AVSSTNS ANCGTCWAVTYPETGVT INV MNTAMCSNGPHGL ASKF P TF GDLPD YPYVGGVF AVSS WNS ANCGTCWAVTYPETGVT INV MNT AACSNGP HGL ASKF P TF GDLP D YP YVGGVF AVSS WNS ANCGTCW AVTYPETGVT INV

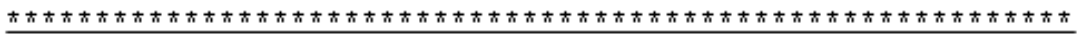

LA IDVASPGFNVAQA AMD KL TNGKA TQLGKVEVNVEQVPTS ACKL LA IDVASPGFNV AQA AMD KL TNGKATQLGKVEVNVEQVPTS ACKL LA IDVASP GFNV AQA AMD KL TNGKA TQLGKVEVNVEQVP TS ACKL LA IDV AS P GFNVAQAMMDKL TNGKA TQL GKVEVNVEQVP TS ACKL

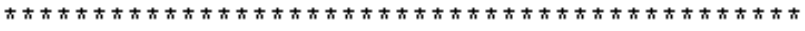


Fig. 2
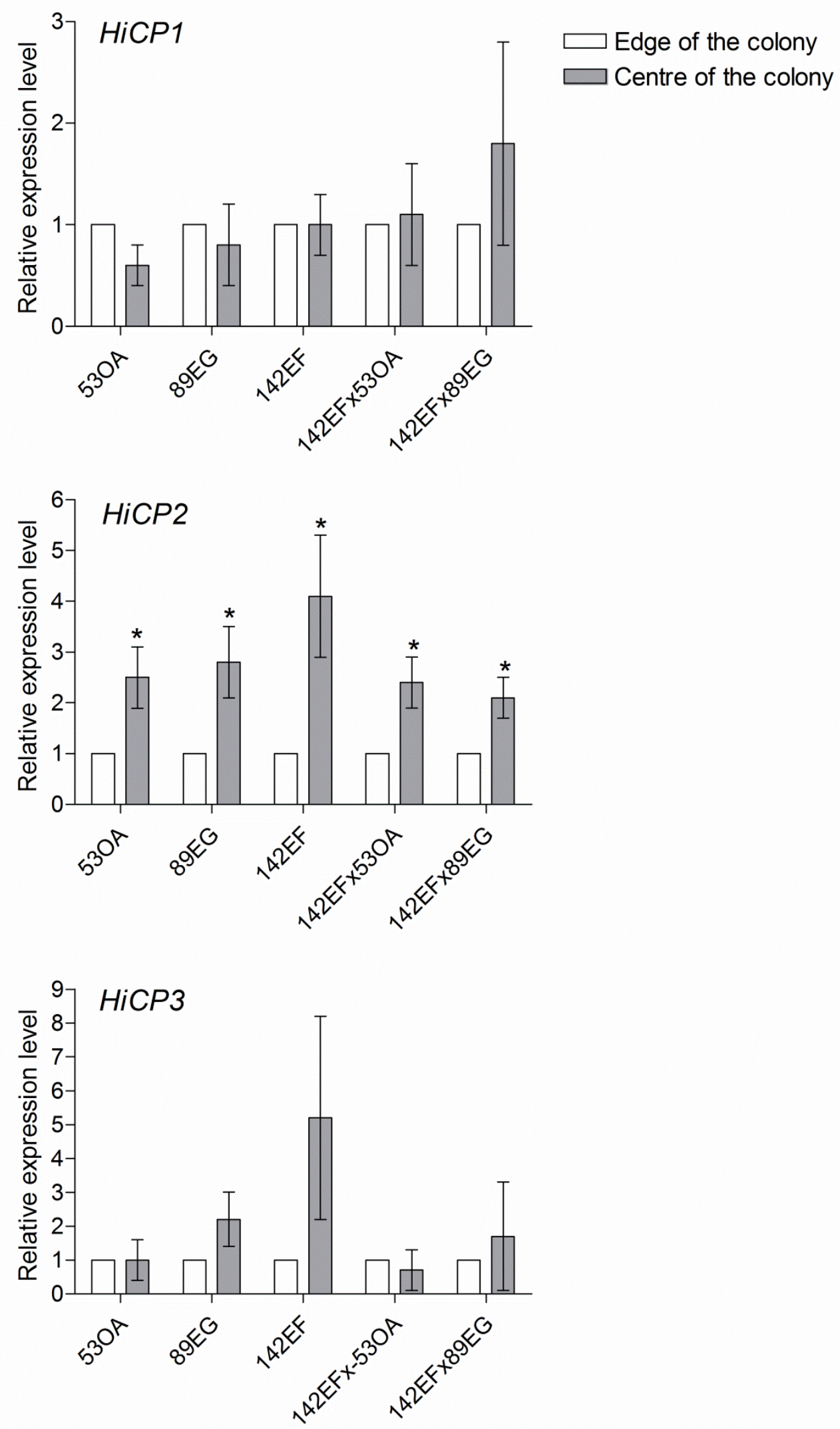
Fig. 3

a
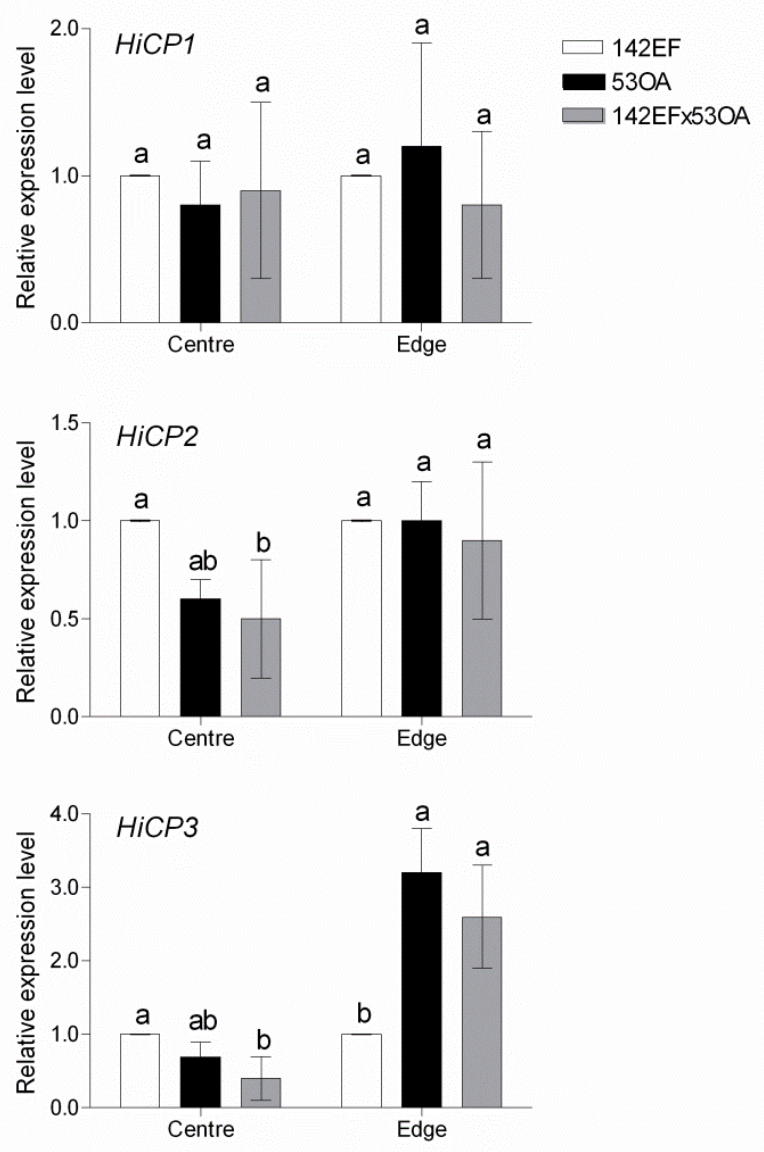

b
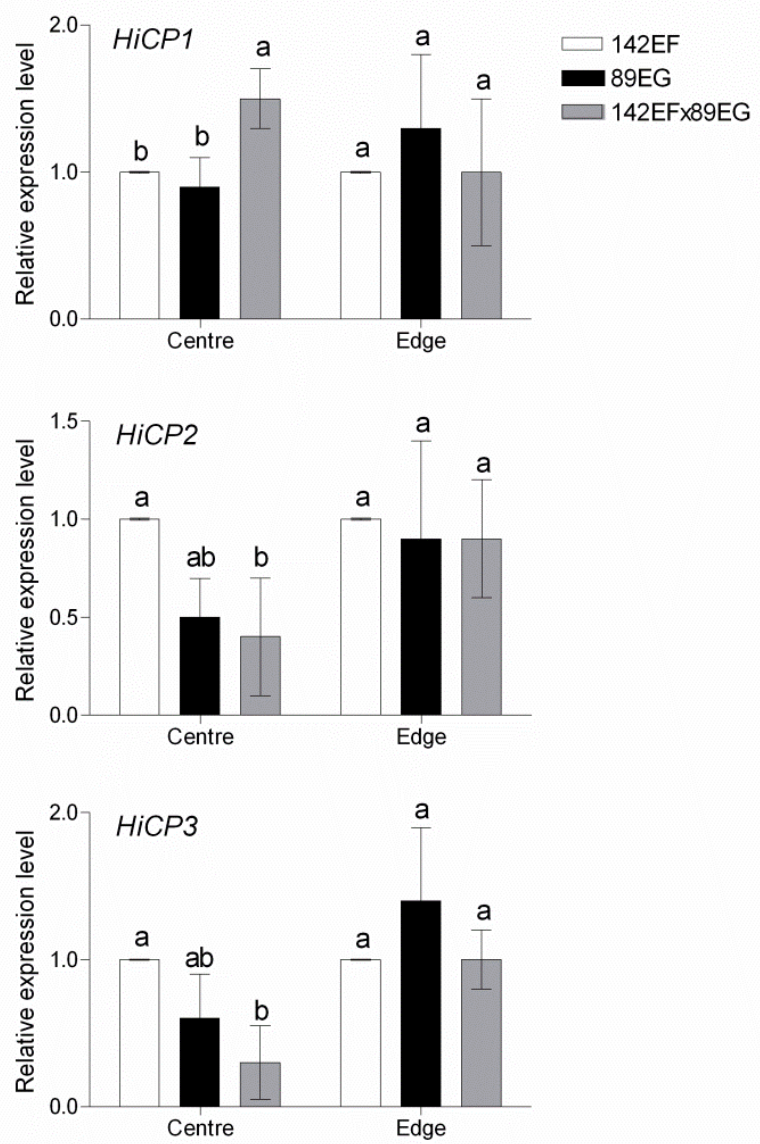

Fig.4

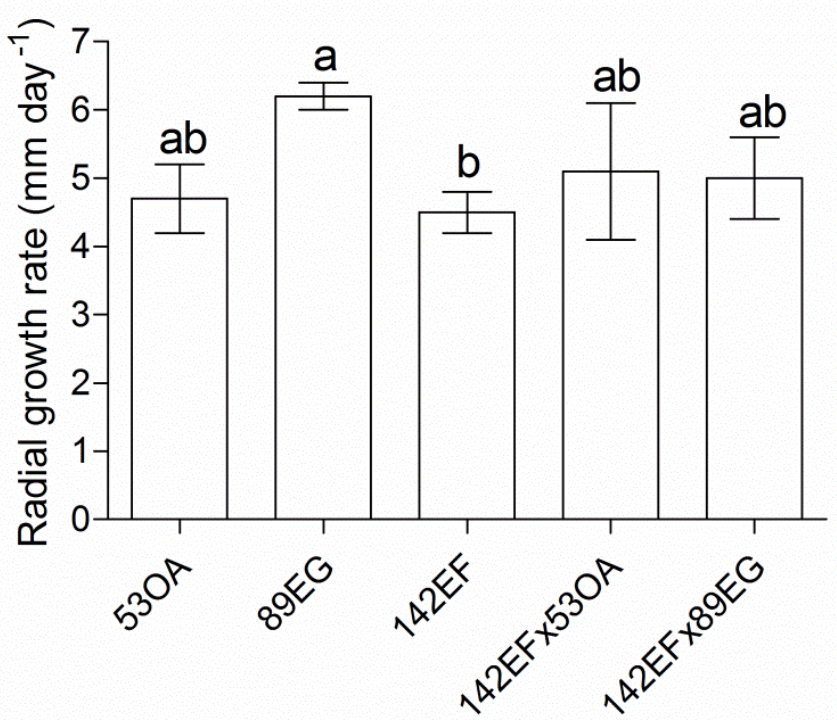


Gene expression analyses reveal a relationship between conidiation and cerato-platanin in homokaryotic and heterokaryotic strains of the fungal plant pathogen Heterobasidion irregulare

Ivan Baccelli ${ }^{1 *}$, Paolo Gonthier ${ }^{2}$, Rodolfo Bernardi ${ }^{3}$

${ }^{1}$ Department of Agri-Food Production and Environmental Sciences, University of Florence, Piazzale delle Cascine 28, 50144, Firenze, Italy; ${ }^{2}$ Department of Agricultural, Forest and Food Sciences, University of Turin, Largo Paolo Braccini 2, 10095, Grugliasco, Italy; ${ }^{3}$ Department of Agriculture, Food and Environment, University of Pisa, Via del Borghetto 80, 56124, Pisa, Italy;

*E-mail: ivan.baccelli@unifi.it (for correspondence) 
Fig. S1 ClustalW alignment of $\mathrm{HiCP} 1$ coding sequences. Partial gene sequences obtained from the Italian isolates in the present study (142EF, 89EG, 53OA) were aligned with the sequence presents in MycoCosm for the American strain TC32-1. Start and stop codons are highlighted in red. Single nucleotide polymorphisms (SNPs) are highlighted in green. Italic underlined indicates the intron

$$
\begin{aligned}
& \text { CP1-TC32-1 } \\
& \text { CP1-53OA } \\
& \text { CP1-142EF } \\
& \text { CP1-89EG }
\end{aligned}
$$

CP1-TC32-1

CP1-530A

$\mathrm{CP} 1-142 \mathrm{EF}$

$\mathrm{CP} 1-89 \mathrm{EG}$

$$
\begin{aligned}
& \text { CP1-TC32-1 } \\
& \text { CP1-53OA } \\
& \text { CP1-142EF } \\
& \text { CP1-89EG }
\end{aligned}
$$

CP1-TC32-1

CP1-530A

$\mathrm{CP} 1-142 \mathrm{EF}$

$\mathrm{CP} 1-89 \mathrm{EG}$

$\mathrm{CP} 1-\mathrm{TC} 32-1$

CP1-530A

CP1-142EF

CP1-89EG

$$
\begin{aligned}
& \text { CP1-TC32-1 } \\
& \text { CP1-53OA } \\
& \text { CP1-142EF } \\
& \text { CP1-89EG }
\end{aligned}
$$

CP1-TC32-1

CP1-530A

CP1-142EF

CP1-89EG

CP1-TC32-1

CP1-530A

CP1-142EF

CP1-89EG

CP1-TC32-1

CP1-530A

CP1-142EF

CP1-89EG

CP1-TC32-1

CP1-530A

CP1-142EF
TCCTATCTCTTTACGTCTGTCCACGACAACCCTCCGTCCACCGACAATGAAGTTCACGTT TCCTATCTCTTTACGTCTGTCCACGACAACCCTCCGTCCACCGACAATGAAGTTCACGTT TCCTATCTCTTTACGTCTGTCCACGACAACCCTCCGTCCACCGACAATGAAGTTCACGTT TCCTATCTCTTTACGTCTGTCCACGACAACCCTCCGTCCACCGACAATGAAGTTCACGTT

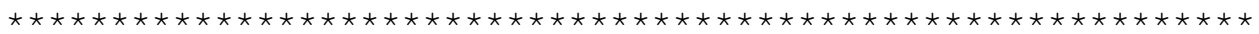

CGCCCTCGCATCCCTCGCCGTTCTCGCATCTACAGCGTTCGCCACTGACGTCCGCTACGA CGCCCTCGCATCCCTCGCCGTTCTCGCATCTACAGCGTTCGCCACTGACGTCCGCTACGA CGCCCTCGCATCCCTCGCCGTTCTCGCATCTACAGCGTTCGCCACTGACGTCCGCTACGA CGCCCTCGCATCCCTCGCCGTTCTCGCATCTACAGCATTCGCCACTGACGTCCGCTACGA

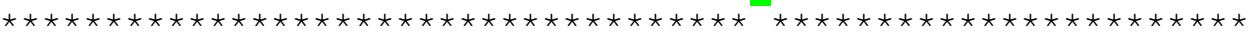

CGAGACTTATGACAACGCCAACGAACCCCTCACGGACGTCGCCTGCTCCGATGGCACGAA CGAGACTTATGACAACGCCAACGAACCCCTCACGGACGTCGCCTGCTCCGATGGCACGAA CGAGACTTATGACAACGCCAACGAACCCCTCACGGACGTCGCCTGCTCCGATGGCACGAA CGAGACTTATGACAACGCCAACGAACCCCTCACGGACGTCGCCTGCTCCGATGGCACGAA

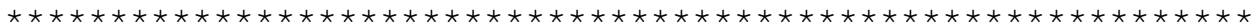

CGGCCTCATCAACAAGGGCTTCAGCACCCTCGGCTCTTTACCGTCTTTCCCGAACGTCGC CGGCCTCATCAACAAGGGCTTCAGCACCCTCGGCTCTTTACCGTCTTTCCCGAACGTCGC CGGCCTCATCAACAAGGGCTTCAGCACCCTCGGCTCTTTACCGTCTTTCCCGAACGTCGC CGGCCTCATCAACAAGGGCTTCAGCACCCTCGGCTCTTTACCGTCTTTCCCGAACGTCGC

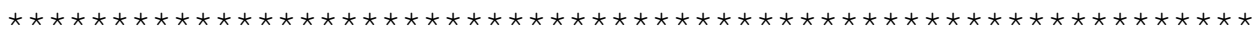

TGCTGTCCAGGCGATTGCCGGGTGGAACTCACCTAGCTGTGGGACTTGCTGGGAGGTCAC TGCTGTCCAGGCGATTGCCGGGTGGAACTCACCTAGCTGTGGGACTTGCTGGGAGGTCAC TGCTGTCCAGGCGATTGCCGGGTGGAACTCACCTAGCTGTGGGACTTGCTGGGAGGTCAC TGCTGTCCAGGCGATTGCCGGGTGGAACTCACCTAGCTGTGGGACTTGCTGGGAGGTCAC

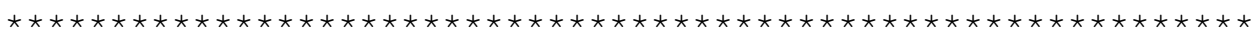

GTACAACGGCCGTAGCGTCCTTGTCACGGGGGTGGATCATGCCGGCGACGGAATCAACAT GTACAACGGCCGTAGCGTCCTTGTCACGGGGGTGGATCATGCCGGCGACGGAATCAACAT GTACAACGGCCGTAGCGTCCTTGTCACGGGGGTGGATCATGCCGGCGACGGAATCAACAT GTACAACGGCCGTAGCGTCCTTGTCACGGGGGTGGATCATGCCGGCGACGGAATCAACAT

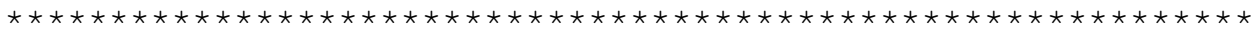

GTCGTTGGAGGCGATGAATACCTTGACGAACAATCAGGGTGTGGCGTTGGGTACTGTGTC GTCGTTGGAGGCGATGAATACCTTGACGAACAATCAGGGTGTGGCGTTGGGTACTGTGTC GTCGTTGGAGGCGATGAATACCTTGACGAACAATCAGGGTGTGGCGTTGGGTACTGTGTC GTCGTTGGAGGCGATGAATACCTTGACGAACAATCAGGGTGTGGCGTTGGGTACTGTGTC

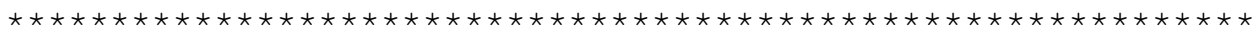

GGCGACAGTAACGCAGGTTGCGGCGTCCCAGTGTGGATTGTAGGGTCAGTTTGGTTCACT GGCGACAGTAACGCAGGTTGCGGCGTCCCAGTGTGGATTGTAGGGTCAGTTTGGTTCACT AGCGACAGTAACGCAGGTTGCGGCGTCCCAGTGTGGATTGTAGGGTGAGTTTGGTTCACT GGCGACAGTAACGCAGGTTGCGGCGTCCCAGTGTGGATTGTAGGGTGAGTTTGGTTCACT

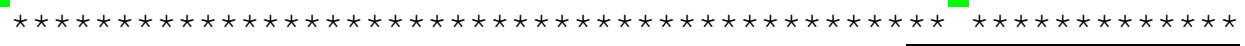

CACAGCTCGCTGTCTGAATCCTTCCTGACTATCTTCAATACCGTTTAAGATCATGCATGA CACAGCTCGCTGTCTGAATCCTTCCTGACTATCTTCAATACCGTTTAAGATCATGCATGA CACAGCTCGCTGTCTGAATCTTTCCTGACTATCTTCAATACCGTTTAAGATCATGCATGA CACAGCTCGCTGTCTGAATCCTTCCTGACCATCTTCAATACCGTTTAAGATCATGCATGA

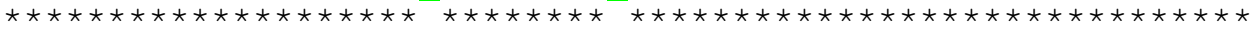


Fig. S2 ClustalW alignment of $\mathrm{HiCP} 2$ coding sequences. Partial gene sequences obtained from the Italian isolates in the present study (142EF, 89EG, 53OA) were aligned with the sequence presents in MycoCosm for the American strain TC32-1. Start and stop codons are highlighted in red. Single nucleotide polymorphisms (SNPs) and the deletion are highlighted in green

$$
\begin{aligned}
& \text { CP2-89EG } \\
& \text { CP2-142EF } \\
& \text { CP2-TC } 32-1 \\
& \text { CP2-530A }
\end{aligned}
$$

$$
\begin{aligned}
& \text { CP2-89EG } \\
& \text { CP } 2-142 \mathrm{EF} \\
& \text { CP } 2-\mathrm{TC} 32-1 \\
& \text { CP2-53OA }
\end{aligned}
$$

$$
\begin{aligned}
& \text { CP2 }-89 \mathrm{EG} \\
& \text { CP2-142EF } \\
& \text { CP2-TC32-1 } \\
& \text { CP2-53OA }
\end{aligned}
$$

$$
\begin{aligned}
& \text { CP2 }-89 E G \\
& \text { CP } 2-142 \mathrm{EF} \\
& \text { CP } 2-\mathrm{TC} 32-1 \\
& \text { CP2-53OA }
\end{aligned}
$$

$$
\begin{aligned}
& \text { CP2-89EG } \\
& \text { CP2-142EF } \\
& \text { CP2-TC32-1 } \\
& \text { CP2-53OA }
\end{aligned}
$$

$$
\begin{aligned}
& \text { CP2 }-89 E G \\
& \text { CP2-142EF } \\
& \text { CP2-TC32-1 } \\
& \text { CP2-53OA }
\end{aligned}
$$

$$
\begin{aligned}
& \text { CP2-89EG } \\
& \text { CP2-142EF } \\
& \text { CP2-TC32-1 } \\
& \text { CP2-53OA }
\end{aligned}
$$

$$
\begin{aligned}
& \text { CP2-89EG } \\
& \text { CP2-142EF } \\
& \text { CP2-TC32-1 } \\
& \text { CP2-53OA }
\end{aligned}
$$

CP2-89EG

$\mathrm{CP} 2-142 \mathrm{EF}$

CP2 2 TC $32-1$

CP $2-530 A$

CP2-89EG

$\mathrm{CP} 2-142 \mathrm{EF}$ CP2-TC32-1
ATTGCTCAACGCACAGCACCCACTCAAGAGGCCCATGCGCGCTCTCGTATTCCTCCTATC ATTGCTCAACGCACAGCACCCACTCAAGAGGCCCATGCGCGCTCTCGTATTCCTCCTATC ATTGCTCAACGCACAGCACCCACTCAAGAGGCCCATGCGCGCTCTCGTATTCCTCCTATC ATTGCTCAACGCACAGCACCCACTCAAGAGGCCCATGCGCGCTCTCGTATTCCTCCTATC

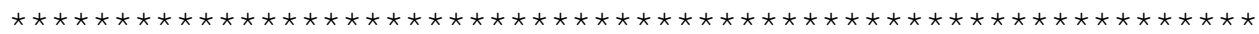

CGTAAATGTGTATAAAAGGGGAGTTGAACTTCGTTTATTTTTCCTTCACCAATTCATTGC CGTAAATGTGTATAAAAGGGGAGTTGAACTTCGTTTATTTTTCCTTCACCAATTCATTGC CGTAAATGTGTATAAAAGGGGAGTTGAACTTCGTTTATTTTTCCTTCACCAATTCATTGC CGTAAATGTGTATAAAGGGGAGTTGAACTTCGTTTATTTTTCCTTCACCAATTTATTGC

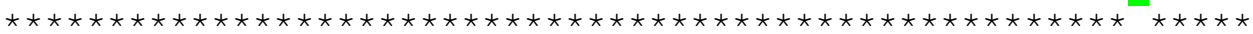

ATTTACACACTCAATAACTACCCAACCAACCATCTATCTGCTATACCACTATTCACCATG ATTTACACACTCAATAACTACCCAACCAACCATCTATCTGCTATACCACTATTCACCATG ATTTACACACTCAATAACTACCCAACCAACCATCTATCTGCTATACCACTATTCACCATG ATTTACACACTCAATAACTACCCAACCAACCATCTATCTGCTATATCACTATTCACCATG

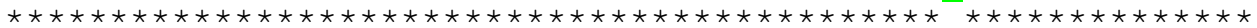

AAGTTCACTGCGTCCTTCATCGCTGTCGCTGCACTCTTCCACGGCACCGCTGCCGCTCCT AAGTTCACTGCGTCCTTCATCGCTGTCGCTGCACTCTTCCACGGCACCGCTGCCGCTCCT AAGTTCACTGCGTCCTTCATCGCTGTCGCTGCACTCTTCCACGGCACCGCTGCCGCTCCT AAGTTTACTGCGTCCTTCATCGCTGTCGCTGCACTCTTCCACGGCACCGCTGCCGCTCCT

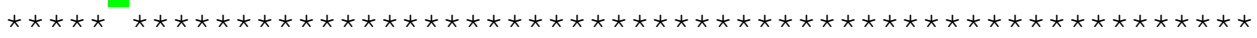

CAGGACGGTGGAGCTTCCCCCACACCCAACAGCCCATCGGGGACGTCTGCGACGTCTGCG CAGGACGGTGGAGCTTCCCCCACACCCAACAGCCCATCGGGGACGTCTGCGACGTCTGCG CAGGACGGTGGAGCTCCCCCCACACCCAACAGCCCATCGGGGACGTCTGCGACGTCTGCG CAGGATGGTGGAGCTCCCCCCACACCCAACAGCCCATCGGGGACGTCTGCGACGTCTGCG

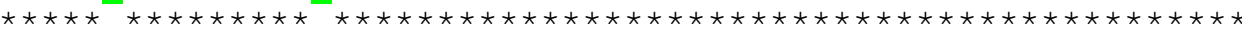

GTGACCTGGTCCAAACCATCTACGTCCCCGGTGTATTCCTCCACCACCTCAACCTACTCA GTGACCTGGTCCAAACCATCTACGTCCCCGGTGTATTCCTCCACCACCTCAACCTACTCA GTGACCTGGTCCAAACCATCTACGTCCCCGGTGTATTCCTCCACCACCTCAACCTACTCA GTGACCTGGTCCAAACCATCTACGTCCCCGGTGTATTCCTCCACCACCTCAACCTACTCA

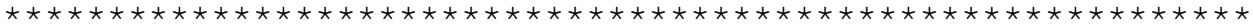

TCTACTACATCGACCACTTCTCCGTATACAACCACCTCCGCCACCACGACATGGTATCCT TCTACTACATCGACCACTTCTCCGTATACAACCACCTCCGCCACCACGACATGGTATCCT TCTACTACATCGACCACTTCTCCGTATACAACCACCTCCGCAACCACGACATGGTATCCT TCTACTACATCGACCACTTCTCCGTATACAACC---TCCGCAACCACGACATGGTATCCT

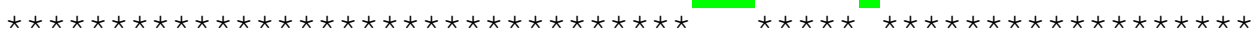

TCGAGTACCTCTTCGTGGTATCCACCGTCCACCTCTTCGAAGCCCTATCCCTCGTCCAGT TCGAGTACCTCTTCGTGGTATCCACCGTCCACCTCTTCGAAGCCCTATCCCTCGTCCAGT TCGAGTACCTCTTCGTGGTATCCACCGTCCACCTCTTCGAAGCCCTATCCCTCGTCCAGT TCGAGTACATCTTCGTGGTATCCACCGTCCACCTCTTCGAAGCCCTATCCCTCGTCCAGT

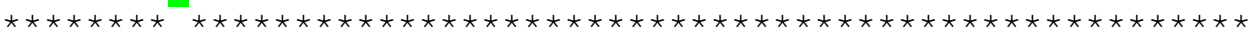

TCATATCCAGTTCCTTCATCGTCCCATTCATATCCCCCTCCCCCTTCGGGTAGTGCTACC TCATATCCAGTTCCTTCATCGTCCCATTCATATCCCCCTCCCCCTTCGGGTAGTGCTACC TCATATCCAGTTCCTTCATCGTCCCATTCATATCCCCCTCCCCCTTCGGGTAGTGCTACC TCATATCCAGTTCCTTCATCGTCCCATTCATATCCCCCTCCCCCTTCGGGTAGTGCTACC

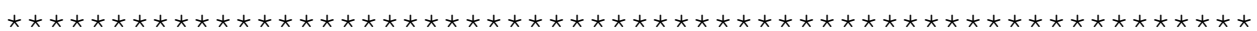




$$
\begin{aligned}
& \text { CP2-530A } \\
& \text { CP2-89EG } \\
& \text { CP2-142EF } \\
& \text { CP2 } 2 \text { TC } 32-1 \\
& \text { CP2 } 2530 A
\end{aligned}
$$

CP2-89EG

$\mathrm{CP} 2-142 \mathrm{EF}$

CP2 2 TC $32-1$

$\mathrm{CP} 2-530 \mathrm{~A}$

$$
\begin{aligned}
& \text { CP2-89EG } \\
& \text { CP2-142EF } \\
& \text { CP2-TC } 22-1 \\
& \text { CP2-53OA }
\end{aligned}
$$

CP2-89EG

$\mathrm{CP} 2-142 \mathrm{EF}$

CP2-TC32-1

CP2 $-530 \mathrm{~A}$

$$
\begin{aligned}
& \text { CP2 }-89 E G \\
& \text { CP } 2-142 \mathrm{EF} \\
& \text { CP } 2-\mathrm{TC} 32-1 \\
& \text { CP } 2-530 A
\end{aligned}
$$

CP2-89EG

$\mathrm{CP} 2-142 \mathrm{EF}$

CP2 $2-\mathrm{TC} 32-1$

CP2 $-530 \mathrm{~A}$

$$
\begin{aligned}
& \text { CP2 }-89 E G \\
& \text { CP2-142EF } \\
& \text { CP2-TC32-1 } \\
& \text { CP2-53OA }
\end{aligned}
$$

$$
\begin{aligned}
& \text { CP2-89EG } \\
& \text { CP2 }-142 \mathrm{EF} \\
& \text { CP } 2-T C 32-1 \\
& \text { CP } 2-530 A
\end{aligned}
$$

CCTCCATACCCGTCAAACTGCCCTCCGAACCCAAATTCCAATCCCCTCATGATGTCGCTT

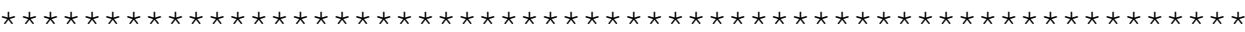

CCTCTTACATATGACAATACGTACGACAATGGGTCTGGTTCTATGAACAGCGTCGCCTGC CCTCTTACATATGACAATACGTACGACAATGGGTCTGGTTCTATGAACAGCGTCGCCTGC ССTCTTACATATGACAATACGTACGACAATGGGTCTGGTTCTATGAACAGCGTCGCTTGC CСTCTTACATATGACAATACGTACGACAATGGGTCTGGTTCTATGAACAGCGTCGCTTGC

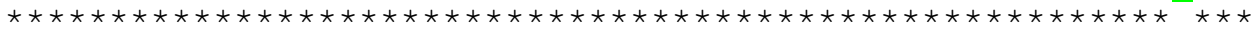

TCCAATGGGCCCAAGGGACTTGTGGGACGCTTCCCGACCTTCAGCGATCTACCCACCTTC TCCAATGGGCCCAAGGGACTTGTGGGACGCTTCCCGACCTTCAGCGATCTACCCACCTTC TCCAATGGGCCCAAGGGACTTGTGGAACGCTTCCCGACCTTCAGCGATCTACCCACCTTC TCCAATGGGCCCAAGGGACTTGTGGAACGCTTCCCGACCTTCAGCGATCTACCCACCTTC

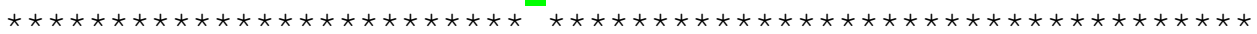

CCCTATATTGGCGGTGCTTTCGCCGTCGGGTCATGGAGCTCGCCTAACTGTGGATCATGC CCCTATATTGGCGGTGCTTTCGCCGTCGGGTCATGGAGCTCGCCTAACTGTGGATCATGC CCCTATATTGGCGGTGCTTTCGCCGTCGGGTCATGGAGCTCGCCTAACTGTGGATCATGC CCCTATATTGGCGGTGCTTTCGCCGTCGGGTCATGGAGCTCGCCTAACTGTGGATCATGC

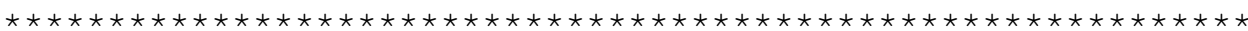

TGGAGTCTCACCTATCCGCAGACCGGCGTCACGATCAAGCTGATCGCTATTGACACATCG TGGAGTCTCACCTATCCGCAGACCGGCGTCACGATCAAGCTGATCGCTATTGACACATCG TGGAGTCTCACCTACCCGCAGACCGGCGTCACGATCAAGCTGATCGCTATTGACACATCG TGGAGTCTCACCTACCCGCAGACCGGCGTCACGATCAAGCTGATCGCTATTGACACATCG

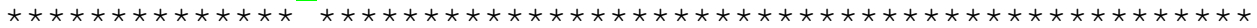

GGCGTTGGTTTCAACGCCGCTCAGGCGGCAATGGACAAGTTGACGGGTGGAAAGGCGAAC GGCGTTGGTTTCAACGCCGCTCAGGCGGCAATGGACAAGTTGACGGGTGGAAAGGCGAAC GGCGTTGGTTTCAACGCCGCTCAGGCGGCAATGGACAAGTTGACGGGTGGAAAGGCGAAC GGCGTTGGTTTCAACGCCGCTCAGGCGGCAATGGACAAGTTGACGGGTGGAAAGGCGAAC

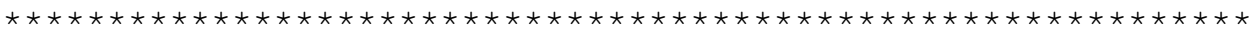

CAGCTTGGTAGGATCGAAGTCAACGCTTACCAGCTTCCTGCCTCGGAGTGCAAGTTGTAG CAGCTTGGTAGGATCGAAGTCAACGCTTACCAGCTTCCTGCCTCGGAGTGCAAGTTGTAG CAGCTTGGTAGGATCGAAGTCAACGCTTACCAGCTTCCTGCCTCGGAGTGCAAGTTGTAG CAGCTTGGTAGGATCGAAGTCAACGCTTACCAGCTTCCTGCCTCGGAGTGCAAGTTGTAG

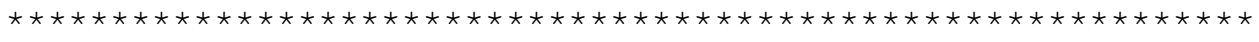

AAAAGCATATTCTCCTTATTTACATTACATTACGGTCACAGATTGATTTTCACATATCAT AAAAGCATATTCTCCTTATTTACATTACATTACGGTCACAGATTGATTTTCACATATCAT AAAAGCATATTCTCCTTATTTACATTACATTACGGTCACAGATTGATTTTCACATATCAT AAAAGCATATTCTCCTTATTTACATTACATTACGGTCACAGATTGATTTTCACATATCAT

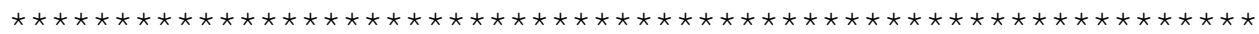

TATATCTTGGATTTC TATATCTTGGATTTC TATATCTTGGATTTC TATATCTTGGATTTC

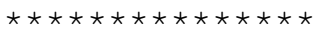


Fig. S3 ClustalW alignment of $\mathrm{HiCP} 3$ coding sequences. Partial gene sequences obtained from the Italian isolates in the present study (142EF, 89EG, 53OA) were aligned with the sequence presents in MycoCosm for the American strain TC32-1. Putative start and stop codons are highlighted in red. Single nucleotide polymorphisms (SNPs) are highlighted in green

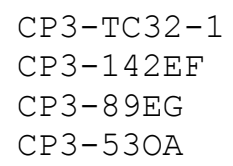

ACGCCAGCAGTTCTACGCCAAGTGCCTACTCGACTTCCGCCGACTCATACCACAGTGAGA ACGCCAGCAGTTCTACGCCAAGTGCCTACTCGACTTCCGCCGACTCATACCACAGTGAGA ACGCCAGCAGTTCTACGCCAAGTGCCTACTCGACTTCCGCCGACTCATACCACAGTGAGA ACGCCAGCAGTTCTACGCCAAGTGCCTACTCGACTTCCGCCGACTCATACCACAGTGAGA

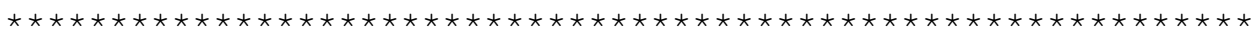

CCTCTTATCCCAGCGGCACGTCATACTCGAGTGAAGCGCCATACCCCTCCCCCTCAAGCA CCTCTTATCCCAGCGGCACGTCATACTCGAGTGAAGCGCCATACCCCTCCCCCTCAAGCA CCTCTTATCCCAGCGGCACGTCATACTCGAGTGAAGCGCCATACCCCTCCCCCTCAAGCA CСTCTTATCCCAGCGGCACGTCATACTCGAGTGAAGCGCCATACCCCTCCCCCTCAAGCA

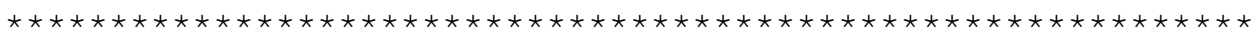

ACTGCCCCTCTTACAATAAACCAGTCTTGACACTTCCCCTGACTTACGACACTGTCTTCG ACTGCCCCTCTTACAATAAACCAGTCTTGACACTTCCCCTGACTTACGACACTGTCTTCG ACTGCCCCTCTTACAATAAACCAGTCTTGACACTTCCCCTGACTTACGACACTGTCTTCG ACTGCCCCTCTTACAATAAACCAGTCTTGACACTTCCCCTGACTTACGACACTGTCTTCG

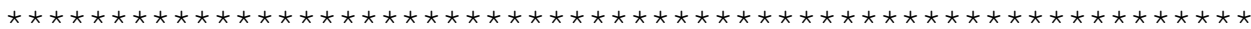

ACAACAAGGCGGGTTCTATGAACACCGCCGCCTGCTCGAATGGCCCTCACGGTCTCGCCT ACAACAAGGCGGGTTCTATGAACACCGCCGCCTGCTCGAATGGCCCTCACGGTCTCGCCT ACAACAAGGCGGGTTCTATGAACACCGCCGCCTGCTCGAATGGCCCTCACGGTCTCGCCT ACAACAAGGCGGGTTCTATGAACACCGCCGCCTGCTCGAATGGCCCTCACGGTCTCGCCT

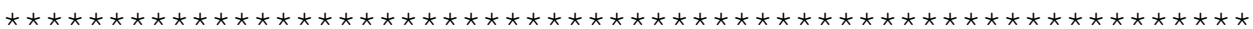

CGAAATTTCCCACATTCGGTGACCTCCCTGACTACCCCTATGTCGGCGGTGTCTTCGCCG CGAAATTTCCCACATTCGGTGACCTCCCTGACTACCCCTATGTCGGCGGTGTCTTCGCCG CGAAATTTCCCACATTCGGTGACCTCCCTGACTACCCCTATGTCGGCGGTGTCTTCGCCG CGAAATTTCCCACATTCGGTGACCTCCCTGACTACCCCTATGTCGGCGGTGTCTTCGCCG

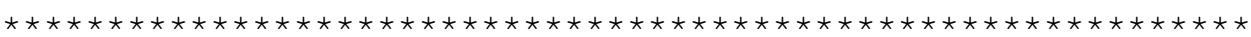

TCTCATCCTGGAACTCTGCCAACTGTGGAACCTGCTGGGCTGTTACATATCCCGAGACTG TCTCATCCTGGAACTCTGCCAACTGTGGAACCTGCTGGGCTGTTACATATCCCGAGACTG TCTCATCCTGGAACTCTGCCAACTGTGGAACCTGCTGGGCTGTTACATATCCCGAGACTG TCTCATCCTGGAACTCTGCCAACTGTGGAACCTGCTGGGCTGTTACATATCCCGAGACTG

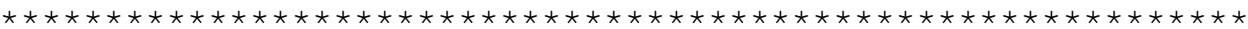

GTGTTACGATCAATGTCCTCGCGATCGATGTGGCCAGCCCCGGTTTCAATGTCGCCCAGG GTGTTACGATCAATGTCCTCGCGATCGATGTGGCCAGCCCCGGTTTCAATGTCGCCCAGG GTGTTACGATCAATGTCCTCGCGATCGATGTGGCCAGCCCCGGTTTCAATGTCGCCCAGG GTGTTACGATCAATGTCCTCGCGATCGATGTGGCCAGCCCCGGTTTCAATGTCGCCCAGG

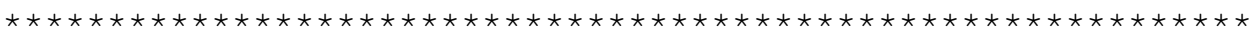

CGGCCATGGACAAGTTGACAAACGGCAAGGCTACTCAGCTCGGAAAGGTCGAGGTCAACG CGGCCATGGACAAGTTGACAAACGGCAAGGCTACTCAGCTCGGAAAGGTCGAGGTCAACG CGGCCATGGACAAGTTGACAAACGGCAAGGCTACTCAGCTCGGAAAGGTCGAGGTCAACG CGGCCATGGACAAGTTGACAAACGGCAAGGCGACTCAGCTCGGAAAGGTCGAGGTCAACG

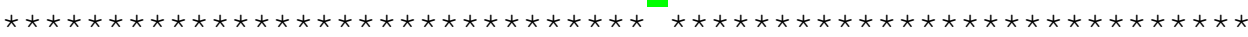

TGGAGCAAGTTCCTACTTCCGCTTGCAAGTTGTGAAAGGTGGGGAGCTGGACTCTCCTTC TGGAGCAAGTTCCTACTTCCGCTTGCAAGTTGTGAAAGGTGGGGAGCTGGACTCTCCTTC TGGAGCAAGTTCCTACTTCCGCTTGCAAGTTGTGAAAGGTGGGGAGCTGGACTCTCCTTC TGGAGCAAGTTCCTACTTCCGCTTGCAAGTTGTGAAAGGTGGGGAGCTGGACTCTCCTTC

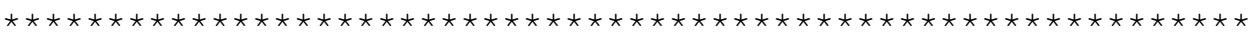


CP3-142EF

CP3-89EG

CP3-530A

CP $3-\mathrm{TC} 32-1$

CP3-142EF

CP $3-89 \mathrm{EG}$

CP3-530A
СTTATACCCCCTTCTTAGAATATACCCTAGTACAGATTGAAATATATTTTTTATTGCTAA CTTATACCCCСTTCTTAGAATATACCCTAGTACAGATTGAAATATATTTTTTATTGCTAA CTTATACCCCCTTCTTAGAATATACCCTAGTACAGATTGAAATATATTTTTTATTGCTAA

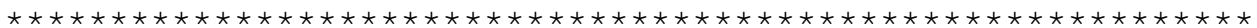

ACATTGGACATGCGCGAAATTGCTATGACTATTTGAAGACATCGGATTCAT ACATTGGACATGCGCGAAATGCTATGACTATTTGAAGACATCGGATTCAT ACATTGGACATGCGCGAAATTGCTATGACTATTTGAAGACATCGGATTCAT ACATTGGACATGCGCGAAATTGCTATGACTATTTGAAGACATCGGATTCAT

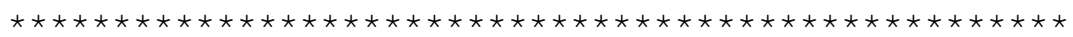


Table S1 Average $\Delta \mathrm{Ct}$ values obtained in the present study per each isolate (centre with edge). $\Delta \mathrm{Ct}$ values were obtained by subtracting the $\mathrm{Ct}$ value of the $18 S$ rRNA gene (reference gene) from the $\mathrm{Ct}$ value of the $C P$ gene (target). The lower the value, the higher the expression.

\begin{tabular}{lccc}
\hline Isolate & $C P 1$ & $C P 2$ & $C P 3$ \\
\hline 53OA & 9,27 & 18,61 & 18,60 \\
89EG & 9,10 & 18,90 & 19,39 \\
142EF & 9,16 & 18,21 & 19,16 \\
142EFx53OA & 9,65 & 18,84 & 19,29 \\
142EFx89EG & 9,01 & 19,02 & 20,11 \\
& & & \\
\hline
\end{tabular}

